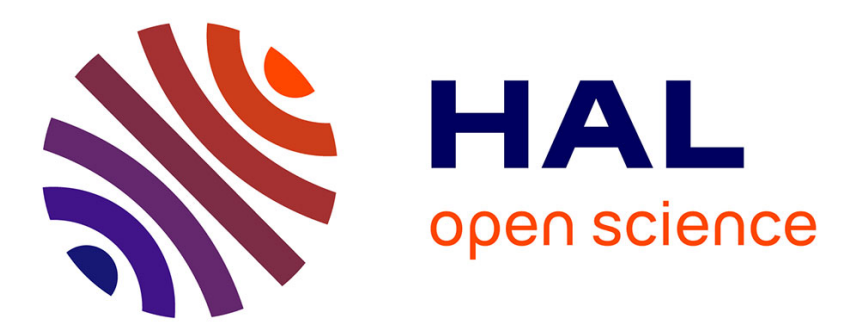

\title{
Autoimmune manifestations associated with myelodysplastic syndromes
}

Eric Grignano, Vincent Jachiet, Pierre P. Fenaux, Lionel Ades, Olivier Fain, Arsene Mekinian

\section{> To cite this version:}

Eric Grignano, Vincent Jachiet, Pierre P. Fenaux, Lionel Ades, Olivier Fain, et al.. Autoimmune manifestations associated with myelodysplastic syndromes. Annals of Hematology, 2018, 97 (11), pp.2015-2023. 10.1007/s00277-018-3472-9 . hal-01960324

\section{HAL Id: hal-01960324 https://hal.sorbonne-universite.fr/hal-01960324}

Submitted on 19 Dec 2018

HAL is a multi-disciplinary open access archive for the deposit and dissemination of scientific research documents, whether they are published or not. The documents may come from teaching and research institutions in France or abroad, or from public or private research centers.
L'archive ouverte pluridisciplinaire HAL, est destinée au dépôt et à la diffusion de documents scientifiques de niveau recherche, publiés ou non, émanant des établissements d'enseignement et de recherche français ou étrangers, des laboratoires publics ou privés. 


\section{Autoimmune manifestations associated with myelodysplastic syndromes}

Eric Grignano ${ }^{1}$, Vincent Jachiet ${ }^{2,3}$, Pierre Fenaux ${ }^{4}$, Lionel Ades ${ }^{4}$, Olivier Fain ${ }^{2,3}$, Arsène Mekinian 2,3

${ }^{1}$ Department of Hematology, Assistance Publique-Hôpitaux de Paris, Hôpital Cochin, 75010 Paris, France

${ }^{2}$ Department of Internal Medicine, Inflammation-Immunopathology-Biotherapy Department (DHU i2B), Assistance Publique - Hôpitaux de Paris, Hôpital Saint-Antoine, 184, rue du Faubourg SaintAntoine, 75012 Paris, France UPMC University Paris 06, Paris, France

${ }^{3}$ Sorbonne Universités, UMPC University Paris 06, INSERM U938, Centre de Recherche Saint-Antoine (CRSA), Paris, France

${ }^{4}$ Department of Hematology, Assistance Publique-Hôpitaux de Paris, Hôpital Saint Louis, 1 avenue Claude Vellefaux, 75010 Paris, France

Correspondence and reprints to Arsène Mékinian, arsene.mekinian@aphp.fr

\section{Conflicts and funding: none}

Running title: Autoimmune diseases in MDS

Key words: Myelodysplastic syndrome, autoimmune disease, hypomethylating agents 


\begin{abstract}
Autoimmune disorders (ADs) are encountered in 10 to $20 \%$ of patients with myelodysplastic syndromes (MDS). Available data suggest that ADs concern more often younger patients with higher-risk IPSS. MDS subtypes associated with ADs are mainly MDS with single lineage dysplasia (MDS-SLD) and MDS with excess blasts (MDS-EB). Various types of ADs have been described in association with MDS, ranging from limited clinical manifestations to systemic diseases affecting multiple organs. Defined clinical entities as vasculitis, connective tissue diseases, inflammatory arthritis and neutrophilic diseases are frequently reported, however, unclassified or isolated organ impairment can be seen. In general, ADs do not seem to confer worse survival, although certain ADs may be associated with adverse outcomes (i.e. vasculitis) or progression of MDS (Sweet syndrome). While steroids and immunosuppressive treatment (IST) remain the backbone of first-line treatment, increasing evidence suggests that MDS specific therapy as hypomethylating agents, based on their immunomodulatory effect, may be effective in treating these complications and for sparing steroids.
\end{abstract}




\section{Introduction}

The myelodysplastic syndromes (MDS) are a group of clonal bone marrow neoplasms characterized by ineffective hematopoiesis, with dysplasia in one or more hematopoietic cell lineages resulting in cytopenia [1]. Natural history is marked by a risk of clonal progression into acute myeloid leukemia (AML).

MDS patients can manifest overt autoimmune disorders (ADs), which pathogenesis remains unknown and the prognostic significance still controversial. ADs associated with MDS have been categorized by Saif et al. into five groups: systemic vasculitis, connective tissue disorders, isolated autoimmune phenomena, immune-mediated hematological abnormalities and asymptomatic serological immunologic abnormalities [2]. The features of these MDS-related disorders seem to be different from idiopathic autoimmune diseases, in particular with more frequent unclassified and incomplete forms [3], [4]. In the presence of features consistent with autoimmune disorder, the physiopathological link between both disorders should be determined, as fortuitous association could be raised.

\section{Epidemiology}

According to the largest retrospective series, ADs can be associated with MDS in 10 to 20\% of cases [4]-[8](Table 1). The prevalence and incidence may vary because of various definitions of ADs among physicians, disparities in patient's selection and the small number of included patients. However, ADs are more frequent in patients with MDS than without MDS (32\% vs 4\%, p<0,001) [9] or those with lymphoid malignancies (5-7\%) [10]. Conversely, autoimmune diseases were associated with an increased risk of AML and MDS [11].

Patients with MDS-associated ADs appear to be younger, with a median age of 66.7-70 years [4], [6] versus 71-73 years in the absence of ADs. The male predominance in MDS patients [1] seem to disappear in MDS-associated ADs [6], [7].

Autoimmune disorders can precede the diagnosis of MDS or occur concomitantly to MDS in $30 \%$ of cases each. In remaining cases ADs develop during the course of MDS with a median time of 8 months [4], but sometimes several years after the MDS onset raising the question of possible fortuitous association [9].

\section{Physiopathology of MDS-related immune dysregulation disorders}


Studies analyzing the physiopathological mechanisms in patients with MDS-related ADs are lacking, although various immune disorders have been described in MDS patients without associated AD. Immune cells changes have been reported in MDS involving NK cells, lymphocytes T-CD4+ and T-CD8+, Treg or B cells, altogether contributing to the defective immune regulation of the clonal malignant cells and progressive disease.

For instance, increased numbers of CD4+ Treg has been associated with unfavorable prognosis of MDS, especially in high-risk patients [12]. The bone marrow recruitment of cytotoxic Tcells and the degree of dyserythropoiesis were inversely related with Treg medullar levels. Blood Treg cells levels were also shown to be an independent prognostic factor in low-risk MDS patients [13]. Treg cells upregulation could explain the impaired cytotoxic anti-tumoral response and thus contribute to malignant cells progression. Strikingly, hypomethylating agents can reverse Treg cells proliferation and immunosuppressive function in high-risk MDS [14]. Usually impaired Treg cells numbers and function have been shown in various systemic autoimmune diseases, and in MDS context could result in an ineffective control of autoreactive cells.

NK cells function have been shown recently to be also impaired in MDS, with inefficient generation of mature and functional NK cells, contributing to the disease progression through impaired immune surveillance [15]. Interestingly, hypomethylating agents can modulate in vitro the killer-cell immunoglobulin-like receptor (KIR) expression at the cell surface in highrisk MDS patients [16], increasing NK cells anti-tumor cell suppression.

B-cell differentiation and progenitors appears to be impaired in low-risk MDS [17]. Hence, more than $50 \%$ of MDS patients generate autoantibodies, mainly directed against autologous erythroblasts, reflecting increased apoptosis related to bone marrow failure, rather than the generation of pathogenic autoantibodies like in idiopathic autoimmune diseases [18]. Interestingly, in some MDS-related ADs, the B-cells depletion induced by rituximab could be effective, particularly in patients with systemic vasculitis [19].

Along with immune cells numbers and functional changes, various cytokines levels have been found to be impaired in MDS patients. Increased TNF $\alpha$ level was observed in MDS patients and correlated with bone marrow cellularity, hemoglobin level and overall survival [20]. While studies using TNF $\alpha$ antagonists have been disappointing in MDS, their efficacy could be raised in some subsets of ADs, in particular MDS-related inflammatory arthritis [19]. 
Altogether these mechanisms result in the escapement of the antitumoral response to the malignant clonal cells, modulating the environment in a favorable layout for the malignant cells expansion. Although the precise mechanisms in MDS-related ADs remain to be determined, the reduced anti-tumoral responses could also be associated with deficient tolerance leading to the emergence of autoreactive cells and immune dysregulation disorders. Further studies are required to highlight the precise mechanisms inducing ADs in MDS patients.

\section{Characteristics of the underlying MDS}

An over-representation of MDS with multilineage dysplasia (MDS-MLD) ( 25\%) and MDS with excess blasts (MDS-EB) ( 23\%) have been shown in a recent case-control study of 788 MDS patients, whereas MDS with single lineage dysplasia (MDS-SLD) ( 9\%) and MDS with ring sideroblasts (MDS-RS) $(\sim 1 \%)$ seem to be less frequent in patients with ADs [4]. In comparison to MDS patients without ADs from the "Groupe Français des Myélodysplasies" (GFM), MDS-EB2 (7\% vs 18\%, p<0,05) and MDS-SB (1\% vs 9\%, p<0,05) were less frequently noted [4]. In another large case-control study, MDS subtypes were MDS-SLD (45.6\%), MDS-MLD (17\%), MDS-EB (28.2\%) and 5q- syndrome (6\%), with similar distribution in MDS patients without ADs, except for MDS-EB (21\% vs 28\%, respectively) [7].

The median levels of hemoglobin, platelets and neutrophils seem to be similar in patients with and without ADs, and higher medullary blast rates have been shown in patients with associated ADs $(9 \%$ vs $5 \%, \mathrm{p}<0,05)[4]$.

\section{Autoimmune disorder characteristics}

Various autoimmune diseases have been described in MDS patients. Among them, systemic diseases have been recently analyzed and classified as vasculitis, connective tissue diseases, inflammatory arthritis, neutrophilic diseases or unclassified disorders. Facing an autoimmune disease, several arguments can raise the hypothesis of an underlying hematological malignancy (Table 2).

In our cohort of 123 patients, vasculitis accounted for $32 \%$ of ADs with predominantly HBVnegative polyarteritis nodosa $(30 \%)$ and giant cell arteritis (23\%). ANCA-negative 
leukocytoclasic vasculitis represents 10 to $30 \%$ of ADs [21], [22]. Behçet's like syndrome, usually with incomplete forms and predominant digestive involvement, may represent up to $15-20 \%$ of cases, especially in MDS with trisomy 8 [4], [23]-[25]. MDS-related vasculitis seem to be associated with more impaired kidney function, more steroid dependency and less complete remission than solid tumor-associated vasculitis [26]. Interestingly, some types of vasculitis have been exceptionally described in primary MDS, like ANCA-associated vasculitis, Takayasu's arteritis or IgA vasculitis, raising very rare association or fortuitous noncausal occurrence.

Connective tissue diseases account for 25-30\% of MDS-related ADs [4], mostly relapsing polychondritis $(\sim 60 \%)$ and systemic lupus erythematosus $(\sim 30 \%)$. Although classical manifestations of relapsing polychondritis are also common in the paraneoplastic form (chondritis and inflammatory arthritis), skin lesions are more frequent, particularly neutrophilic dermatosis [27] [28], [29]. Other connective tissue diseases such as Sjögren's syndrome (SS) or myositis have been exceptionally reported, and no case of systemic sclerosis have been reported [6], [22].

Inflammatory arthritis is another subset of MDS-related ADs. Among them, polymyalgia rheumatica account for $\sim 10 \%$, sometimes associated with giant cell arteritis [6], [30], [31]. Compared to patients without MDS, patients with inflammatory arthritis tend to be older, more often male with more involved joints [32]. Remitting seronegative symmetrical synovitis with pitting edema (RS3PE syndrome) associated with MDS is characterized by more frequent steroids dependence [4], [33]. Undifferentiated arthritis represents the most frequent subset and typically present as polyarticular and symmetrical arthritis, usually without structural progression [4].

Neutrophilic dermatosis, i.e. Sweet syndrome and pyoderma gangrenosum, are common and typical paraneoplastic diseases whose onset must warn on the occurrence of solid tumor or hematological disease. Sweet syndrome is characterized by the presence of inflammatory papules, plaques, or nodules on the skin, systemic symptoms (fever, malaise, joint pain, and muscle pain) and neutrophilic infiltration of the skin without vasculitis. Sweet syndrome approximately accounts for $\sim 10-15 \%$ of ADs [34] and can be induced by G-CSF use or chemotherapy [35]. Neutrophilic dermatosis could be associated with 5q- syndrome and is associated with a worse prognosis of the underlying hemopathy [34]. 
Autoimmune cytopenias in MDS context are rather infrequent. Autoimmune hemolytic anemia (AIHA) have been reported with MDS in approximately 3\% [36], although erythrocytic autoantibodies can be founded in up to $35 \%$ of MDS patients [37]. AIHA is more likely to arise along with a low-risk MDS, as MDS-SLD or MDS-RS [24]. Immune thrombocytopenic purpura (ITP) is also rare with few case reports [38].

Besides these subsets, various systemic or organ specific diseases have been reported in MDS patients, like pulmonary alveolar proteinosis. The proteinosis could be associated with particular karyotype (i.e. dicentric isochrosome of deleted 20q) and seem to be associated with a poor outcome [39]. Few cases of inflammatory bowel disease, especially Crohn disease, is also reported with MDS [40].

\section{Biological immune abnormalities in MDS patients without clinical ADs}

Autoantibodies (AAb) have been found in 53\% of MDS patients without any clinical signs of autoimmune disorder [41]: anti-nuclear antibodies (ANA) without any specificity (20\%), antiphospholipid antibodies (22\%), ANCA without any specificity (9\%), rheumatoid factor $(12 \%)$ and anti-tissue antibodies (13\%). In comparison to age-paired patients without MDS, similar frequency of autoantibodies has been found, except for anti-parietal cells AAb ( $\mathrm{p}=0.05)$. ANCA prevalence was found similar to the general population (3\%) in another case control study, and seem to be useless for the diagnosis of MDS-related vasculitis [42]. Direct antiglobulin test (DAT) positivity is reported in 5-15\% of cases [8], [43].

In comparison to patients without clinical ADs, higher CRP levels and ANA titers have been shown in patients with ADs [41]. However, when patients were treated with hypomethylating agents, they had significantly less often various autoantibodies ( $29 \%$ vs $53 \%$; $\mathrm{p}=0.07)$.

In most cases, autoantibodies screening remain negative, as several MDS-related diseases usually have no specific autoantibodies (p.e polyarteritis nodosa, giant cell arteritis, relapsing polychondritis or polymyalgia rheumatica).

\section{Prognosis}

The prognostic significance of MDS-associated ADs remains controversial. In part, this can be due to the heterogeneity of autoimmune diseases associated with MDS and the fact that in these 
studies, autoimmunity is mostly considered as one phenomenon regardless of the type of autoimmunity. Therefore, depends on types of autoimmune diseases in each cohort, the impact on survival could be different. In 123 patients with ADs and MDS, compared to 665 MDS patients, median overall survival was 72 and 75 months, respectively $(\mathrm{p}=0.2)$, with similar rates of acute myeloid leukemia and deaths. Similarly, no difference in overall survival has been shown in the study by De Hollanda et al, except in the presence of cryoglobulinemic vasculitis [7]. In another prospective study of 70 patients, median overall survival was 39 months in MDS-related ADs patients $(n=13)$ versus 26 months $(n=57)(p=0,76)$ [6]. Several small reports showed that systemic vasculitis could be associated with MDS prognosis, mostly because of the presence of high-risk MDS features [7], [44], [45]. Nevertheless, in cases of polyarteritis nodosa associated with MDS death can be due to the complication of gastrointestinal involvement, with perforations and sepsis [45]. Concerning MDS cytogenetic features, karyotype distribution does not appear to be influenced by the presence of ADs. The rates of favorable cytogenetic findings (normal karyotype 5q-, 20q- or $-\mathrm{Y}$ ) do not differ between MDS patients with or without ADs [4], [6], [7].

A slightly higher percentage of medullary blasts was found in patients with associated ADS $(9 \%$ versus $5 \%)(p<0.05)$ [4], [6]. Using multivariate analysis of factors associated with overall survival, IPSS scale remained the only factor associated with survival similarly to patients without ADs [4].

\section{Treatment of MDS-related ADs}

Treatment of MDS-related ADs could be challenging because of the underlying cytopenias, the risk of infectious complications and secondary MDS [46]. Therapeutic indication and treatment regimens must take account of the subtype of ADs, the severity of the disease, the relapse rates, and the need for specific hematological treatment for the underlying MDS (Figure 1).

Steroids are the most used drugs in first line and induce remission in 80 to $90 \%$ of cases [3], [4]. However, despite initial efficacy, steroid dependence or relapse occurs in $50 \%$ to $70 \%$ of cases, leading to second line treatment, especially in case of vasculitis [26]. While response to the first line occurs in $85 \%$ of cases, only $60 \%$ of patients experiencing second-line treatment has sustained response [4] 
In steroid-dependent or refractory patients or life-threatening disease at onset, other immunosuppressive or immunomodulating drugs including DMARDs (methotrexate, azathioprine, mycophenolate mofetil, cyclophosphamide), biological targeting agents (rituximab, anti-TNFa, tocilizumab and anakinra) should be considered. But the hematological toxicity of methotrexate and cyclophosphamide should limit their use in these conditions.

Few reports showed interesting efficacy of biological targeting drugs, but in a French study of 29 patients biological targeted drugs have shown poor efficacy, in comparison to non-MDS related ADs [19]. Among them, Rituximab was the most effective to induce response (65\%), whereas other treatments such as anti-TNF $\alpha$ antibodies or anti-interleukins antibodies (tocilizumab and anakinra) induced a response in only 1/3 of cases. Rituximab had better response in vasculitis and TNF $\alpha$ antagonists in arthritis. Biological-targeted drugs in MDS patients have been associated with high rates of infection (30\%) and infusion-related side effects, especially for anakinra [19].

\section{Inflammatory arthritis}

In inflammatory arthritis, steroids are usually efficient, particularly in the initial course of the disease. Most frequent steroid dependence has been noted in MDS-related arthritis and may argue for steroid sparing drugs combination. In undifferentiated rheumatism and RS3PE syndrome, corticosteroid therapy may be associated with sulfasalazine or hydroxychloroquine, which can even be used without steroids in mild forms. In MDS patients DMARDs should be avoided, and salazopyrine-hydroxychloroquine combination or TNF $\alpha$ antagonists, tocilizumab or rituximab use may be considered similarly to non-MDS rheumatoid arthritis. In steroid dependent or refractory polymyalgia rheumatica, tocilizumab may be proposed [47].

\section{Skin involvement}

In patients with neutrophilic dermatosis such as Sweet syndrome, steroids are particularly efficient inducing a prompt and complete response in almost all cases. However, steroid dependence is still frequent, requiring the use of thalidomide, dapsone or biological targeted drugs. In isolated leukocytoclasic vasculitis, colchicine or dapsone could be used in mild forms and steroids in patients with more severe disease.

\section{Connective tissue disease}

In patients with systemic lupus erythematosus, low-dose steroids should be associated with hydroxychloroquine, whether thalidomide seems to be efficient in refractory forms. In 
relapsing polychondritis, steroids are highly effective to control the disease, but again steroid dependence is frequent and biological targeted drugs as anti-TNF $\alpha$ antagonists may be considered.

\section{Systemic vasculitis}

In patients with systemic vasculitis in the absence of MDS, therapeutic strategy is usually tailored by the five-factor score (FFS score)[48], in particular in polyarteritis nodosa and ANCA-positive vasculitis. Although no data are available about the value of this scale in MDS-related vasculitis, the need for combined treatment should be discussed similarly to non-MDS vasculitis. In FFS scale $>1$ patients, rituximab could be associated to induce sustained remission. In giant cell arteritis, the steroid dependence and relapse seem to be similar to the idiopathic forms, and thus steroids alone should be used firstly. Tocilizumab may be added in the case of steroid dependence or frequent relapses [49]. In Behçet's like syndrome, associated treatment, as TNF $\alpha$ antagonists should be considered in addition with steroids because of frequent gastrointestinal complications.

\section{Hypometylating agents (HMA)}

Azacytidine significantly improves survival in higher risk MDS [50] and is also approved for acute myeloid leukemia in patients with blasts $>30 \%$ ineligible for intensive regimens [51].

Azacytidine efficacy in MDS-related ADs was reported in few case reports, mainly with refractory MDS-related ADs [52]-[55]. Among 22 patients [56], half of treated patients had int-2 or high IPSS and the remaining had low or int-1 IPSS. In low-risk patients, azacytidine was initiated for transfusion dependence, severe thrombocytopenia or refractory ADs. At azacytidine initiation, 15 patients had uncontrolled ADs. Azacytidine induced complete or partial ADs response in $87 \%$. Steroids tapering were allowed in $63 \%$ and only 3 patients relapsed under treatment. ADs response was obtained after a median of 3 cycles of azacytidine.

The potential efficacy of hypomethylating agents in autoimmune phenomena associated with MDS may be supported by immunomodulatory effects on various immune cells as CD4+ Tcells [53], Treg lymphocytes [14], especially in the context of post-allogeneic stem cells transplant [57], NK cells [16], and pro-inflammatory cytokines modulation, in particular IL-6 [58]. 
To confirm these preliminary data, a non-randomized trial has been recently initiated on behalf of the French MDS network ("Groupe Francophone des Myélodysplasies), addressing the efficacy of azacytidine in ADs associated with MDS (NCT02985190).

\section{Bone marrow engraftment}

Few studies report cases of severe or refractory autoimmune diseases in response after hematopoietic stem cell transplantation, whether in MDS-related context [59] or not [60].

\section{Conclusion and perspectives}

Autoimmune disorders are commonly encountered in MDS patients with various subtypes and unclassified forms. The understanding of common pathways linking MDS and autoimmunity are growing with identification of deregulation in innate and adaptive immune cells, cytokine secretion and genetic landscape underlying MDS. Although the presence of ADs does not impact neither overall survival nor progression to acute leukemia, their management is crucial to avoid steroid dependence and associated adverse events. Increasing evidence suggests that MDS specific therapy as azacytidine, based on their immunomodulatory effect, may be effective in treating ADs and sparing steroids concomitantly to MDS disease control. Prospective studies are ongoing to address the place of azacytidine in such situations and to help physicians in choosing the best therapeutic strategy.

\section{Tables and Figures:}

Table 1: Literature studies of ADs associated with MDS.

Table 2: Arguments for MDS-related ADs in patients with extra-hematological features.

Figure 1: Therapeutic strategy in AID associated MDS. 


\section{Declarations}

*Ethics approval and consent to participate: not applicable.

*Consent for publication: not applicable.

*Availability of data and material: data sharing not applicable to this article as no datasets were generated or analysed during the current study

*Competing interests: the authors declare that they have no competing interests. 
*Funding: none.

\section{*Authors' contributions}

- EG performed the research

- EG and AM designed the research study

- VJ and AM contributed essential reagents or tools

- PF, LA and OF revised the manuscript.

Acknowledgments: none.

\section{References}

[1] L. Adès, R. Itzykson, and P. Fenaux, "Myelodysplastic syndromes," Lancet, Mar. 2014.

[2] M. W. Saif, J. L. Hopkins, and S. D. Gore, "Autoimmune phenomena in patients with myelodysplastic syndromes and chronic myelomonocytic leukemia," Leukemia \& lymphoma, vol. 43, no. 11, pp. 2083-92, Nov. 2002.

[3] H. Enright and W. Miller, "Autoimmune phenomena in patients with myelodysplastic syndromes," Leukemia \& lymphoma, vol. 24, no. 5-6, pp. 483-9, Feb. 1997.

[4] A. Mekinian et al., "Systemic inflammatory and autoimmune manifestations associated with myelodysplastic syndromes and chronic myelomonocytic leukaemia: a French multicentre retrospective study," Rheumatology (Oxford), Sep. 2015.

[5] M. Castro, D. L. Conn, W. P. Su, and J. P. Garton, "Rheumatic manifestations in myelodysplastic syndromes," J. Rheumatol., vol. 18, no. 5, pp. 721-727, May 1991.

[6] S. Giannouli, M. Voulgarelis, E. Zintzaras, A. G. Tzioufas, and H. M. Moutsopoulos, "Autoimmune phenomena in myelodysplastic syndromes: a 4-yr prospective study," Rheumatology (Oxford, England), vol. 43, no. 5, pp. 626-32, May 2004. 
[7] A. de Hollanda et al., "Systemic and immune manifestations in myelodysplasia: a multicenter retrospective study," Arthritis care \& research, vol. 63, no. 8, pp. 1188-94, Aug. 2011.

[8] T. Okamoto et al., "Correlation between immunological abnormalities and prognosis in myelodysplastic syndrome patients," Int. J. Hematol., vol. 66, no. 3, pp. 345-351, Oct. 1997.

[9] S. Berthier, N. Magy, H. Gil, M. Becker Schneider, D. A. Vuitton, and J. L. Dupond, "Myélodysplasies et maladies systémiques. Une association non fortuite," La Revue de Médecine Interne, vol. 22, no. 5, pp. 428-432, May 2001.

[10] E. Grignano, A. Mekinian, V. Jachiet, P. Coppo, and O. Fain, "[Autoimmune and inflammatory disorders associated with lymphoid hematological malignancies]," Rev Med Interne, vol. 38, no. 6, pp. 374-382, Jun. 2017.

[11] L. A. Anderson, R. M. Pfeiffer, O. Landgren, S. Gadalla, S. I. Berndt, and E. A. Engels, "Risks of myeloid malignancies in patients with autoimmune conditions," Br. J. Cancer, vol. 100, no. 5, pp. 822-828, Mar. 2009.

[12] S. Y. Kordasti et al., "CD4+CD25high Foxp3+ regulatory T cells in myelodysplastic syndrome (MDS)," Blood, vol. 110, no. 3, pp. 847-850, Aug. 2007.

[13] A. W. Mailloux et al., "Expansion of effector memory regulatory T cells represents a novel prognostic factor in lower risk myelodysplastic syndrome," J. Immunol., vol. 189, no. 6, pp. 3198-3208, Sep. 2012.

[14] B. Costantini et al., "The effects of 5-azacytidine on the function and number of regulatory T cells and T-effectors in myelodysplastic syndrome," Haematologica, vol. 98, no. 8, pp. 1196-1205, Aug. 2013.

[15] M. Hejazi et al., "Impaired cytotoxicity associated with defective natural killer cell differentiation in myelodysplastic syndromes," Haematologica, vol. 100, no. 5, pp. 643652, May 2015.

[16] E. Sohlberg, A. Pfefferle, S. Andersson, B. C. Baumann, E. Hellström-Lindberg, and K.-J. Malmberg, "Imprint of 5-azacytidine on the natural killer cell repertoire during systemic treatment for high-risk myelodysplastic syndrome," Oncotarget, vol. 6, no. 33, pp. 34178-34190, Oct. 2015.

[17] A. Sternberg et al., "Evidence for reduced B-cell progenitors in early (low-risk) myelodysplastic syndrome," Blood, vol. 106, no. 9, pp. 2982-2991, Nov. 2005.

[18] W. Barcellini et al., "Anti-erythroblast autoimmunity in early myelodysplastic syndromes," Haematologica, vol. 92, no. 1, pp. 19-26, Jan. 2007.

[19] A. Mekinian et al., "Biologics in myelodysplastic syndrome-related systemic inflammatory and autoimmune diseases: French multicenter retrospective study of 29 patients," Autoimmunity Reviews, vol. 16, no. 9, pp. 903-910, Sep. 2017.

[20] G. Stifter, S. Heiss, G. Gastl, A. Tzankov, and R. Stauder, "Over-expression of tumor necrosis factor-alpha in bone marrow biopsies from patients with myelodysplastic syndromes: relationship to anemia and prognosis," Eur. J. Haematol., vol. 75, no. 6, pp. 485-491, Dec. 2005.

[21] M. Dalamaga, K. Karmaniolas, A. Matekovits, I. Migdalis, and E. Papadavid, "Cutaneous manifestations in relation to immunologic parameters in a cohort of primary myelodysplastic syndrome patients," J Eur Acad Dermatol Venereol, vol. 22, no. 5, pp. 543-548, May 2008.

[22] O. A. Ustwani et al., "Myelodysplastic syndromes and autoimmune diseases-Case series and review of literature," Leukemia research, May 2013.

[23] J. K. Ahn et al., "Behcet's disease associated with bone marrow failure in Korean patients: clinical characteristics and the association of intestinal ulceration and trisomy 8," Rheumatology (Oxford), vol. 47, no. 8, pp. 1228-1230, Aug. 2008. 
[24] O. Fain, T. Braun, J. Stirnemann, and P. Fenaux, "[Systemic and autoimmune manifestations in myelodysplastic syndromes]," La Revue de medecine interne / fondee ... par la Societe nationale francaise de medecine interne, vol. 32, no. 9, pp. 552-9, Sep. 2011.

[25] T. Toyonaga et al., "Refractoriness of intestinal Behçet's disease with myelodysplastic syndrome involving trisomy 8 to medical therapies - our case experience and review of the literature," Digestion, vol. 88, no. 4, pp. 217-221, 2013.

[26] O. Fain et al., "Vasculitides associated with malignancies: analysis of sixty patients," Arthritis and rheumatism, vol. 57, no. 8, pp. 1473-80, Dec. 2007.

[27] J. Dion et al., "Relapsing Polychondritis Can Be Characterized by Three Different Clinical Phenotypes: Analysis of a Recent Series of 142 Patients," Arthritis \& Rheumatology (Hoboken, N.J.), vol. 68, no. 12, pp. 2992-3001, Dec. 2016.

[28] B. Myers, J. Gould, and G. Dolan, "Relapsing polychondritis and myelodysplasia: a report of two cases and review of the current literature," Clinical \& Laboratory Haematology, vol. 22, no. 1, pp. 45-48, Feb. 2000.

[29] J. C. Piette, T. Papo, P. Chavanon, D. L. Huong, C. Frances, and P. Godeau, "Myelodysplasia and relapsing polychondritis," J. Rheumatol., vol. 22, no. 6, pp. 12081209, Jun. 1995.

[30] G. Espinosa, J. Font, F. J. Muñoz-Rodríguez, R. Cervera, and M. Ingelmo, "Myelodysplastic and myeloproliferative syndromes associated with giant cell arteritis and polymyalgia rheumatica: a coincidental coexistence or a causal relationship?," Clin. Rheumatol., vol. 21, no. 4, pp. 309-313, Aug. 2002.

[31] A. Mekinian et al., "Inflammatory arthritis in patients with myelodysplastic syndromes: a multicenter retrospective study and literature review of 68 cases," Medicine (Baltimore), vol. 93, no. 1, pp. 1-10, Jan. 2014.

[32] M. Bellan et al., "Association between rheumatic diseases and cancer: results from a clinical practice cohort study," Intern Emerg Med, vol. 12, no. 5, pp. 621-627, Aug. 2017.

[33] S. Paira, C. Graf, S. Roverano, and J. Rossini, "Remitting seronegative symmetrical synovitis with pitting oedema: a study of 12 cases," Clin. Rheumatol., vol. 21, no. 2, pp. 146-149, May 2002.

[34] S. J. Lee et al., "Certain Autoimmune Manifestations Are Associated With Distinctive Karyotypes and Outcomes in Patients With Myelodysplastic Syndrome: A Retrospective Cohort Study," Medicine (Baltimore), vol. 95, no. 13, p. e3091, Mar. 2016.

[35] A. G. Kulasekararaj, S. Kordasti, T. Basu, J. R. Salisbury, G. J. Mufti, and A. W. P. du Vivier, "Chronic relapsing remitting Sweet syndrome--a harbinger of myelodysplastic syndrome," Br. J. Haematol., vol. 170, no. 5, pp. 649-656, Sep. 2015.

[36] F. Van Rhee and M. Abela, "Coombs negative haemolytic anaemia responding to intravenous immunoglobulins in a patient with myelodysplastic syndrome," Clin Lab Haematol, vol. 13, no. 1, pp. 99-101, 1991.

[37] M. C. Novaretti, C. R. Sopelete, E. R. Velloso, M. F. Rosa, P. E. Dorlhiac-Llacer, and D. A. Chamone, "Immunohematological findings in myelodysplastic syndrome," Acta Haematol., vol. 105, no. 1, pp. 1-6, 2001.

[38] S.-J. Park et al., "Cyclosporine A in the treatment of a patient with immune thrombocytopenia accompanied by myelodysplastic syndrome and nephrotic syndrome," Acta Haematol., vol. 110, no. 1, pp. 36-40, 2003.

[39] Y. Xue et al., "Pulmonary alveolar proteinosis as a terminal complication in a case of myelodysplastic syndrome with idic(20q-)," Acta Haematol., vol. 123, no. 1, pp. 55-58, 2010. 
[40] Z. Wang, Y. Zhou, and Y. Liu, "Concurrent inflammatory bowel disease and myelodysplastic syndrome: report of nine new cases and a review of the literature," Dig. Dis. Sci., vol. 53, no. 7, pp. 1929-1932, Jul. 2008.

[41] J. B. Fraison et al., "279 FREQUENCY OF AUTOANTIBODIES (AAB) IN MDS WITH AND WITHOUT CLINICAL AUTOIMMUNE DISORDERS (AID)," Leukemia Research, vol. 39, pp. S139-S140, Apr. 2015.

[42] M. A. Hamidou, S. Derenne, M. A. Audrain, J. M. Berthelot, A. Boumalassa, and J. Y. Grolleau, "Prevalence of rheumatic manifestations and antineutrophil cytoplasmic antibodies in haematological malignancies. A prospective study," Rheumatology (Oxford), vol. 39, no. 4, pp. 417-420, Apr. 2000.

[43] D. Marisavljević, N. Kraguljac, and Z. Rolović, "Immunologic abnormalities in myelodysplastic syndromes: clinical features and characteristics of the lymphoid population," Med. Oncol., vol. 23, no. 3, pp. 385-391, 2006.

[44] E. Grignano et al., "Autoimmune and inflammatory diseases associated with chronic myelomonocytic leukemia: A series of 26 cases and literature review," Leukemia Research, vol. 47, pp. 136-141, Aug. 2016.

[45] M. A. Hamidou, A. Boumalassa, C. Larroche, D. E. Kouri, O. Blétry, and J.-Y. Grolleau, "Systemic medium-sized vessel vasculitis associated with chronic myelomonocytic leukemia," Seminars in Arthritis and Rheumatism, vol. 31, no. 2, pp. 119-126, Oct. 2001.

[46] N. Ertz-Archambault et al., "Association of Therapy for Autoimmune Disease With Myelodysplastic Syndromes and Acute Myeloid Leukemia," JAMA Oncol, vol. 3, no. 7, pp. 936-943, Jul. 2017.

[47] V. Devauchelle-Pensec et al., "Efficacy of first-line tocilizumab therapy in early polymyalgia rheumatica: a prospective longitudinal study," Ann. Rheum. Dis., vol. 75, no. 8, pp. 1506-1510, Aug. 2016.

[48] L. Guillevin et al., "The Five-Factor Score revisited: assessment of prognoses of systemic necrotizing vasculitides based on the French Vasculitis Study Group (FVSG) cohort," Medicine (Baltimore), vol. 90, no. 1, pp. 19-27, Jan. 2011.

[49] A. Régent et al., "Tocilizumab in Giant Cell Arteritis: A Multicenter Retrospective Study of 34 Patients," J. Rheumatol., vol. 43, no. 8, pp. 1547-1552, Aug. 2016.

[50] P. Fenaux et al., "Efficacy of azacitidine compared with that of conventional care regimens in the treatment of higher-risk myelodysplastic syndromes: a randomised, open-label, phase III study," The Lancet Oncology, vol. 10, no. 3, pp. 223-232, Mar. 2009.

[51] H. Dombret et al., "International phase 3 study of azacitidine vs conventional care regimens in older patients with newly diagnosed AML with \&gt;30\% blasts," Blood, vol. 126, no. 3, pp. 291-299, Jul. 2015.

[52] O. Al Ustwani, J. Francis, P. K. Wallace, J. Ambrus Jr., and M. Wetzler, "Treating myelodysplastic syndrome improves an accompanying autoimmune disease along with a reduction in regulatory T-cells," Leukemia research, vol. 35, no. 5, pp. e35-6, May 2011.

[53] J. J. Frietsch et al., "Paraneoplastic inflammation in myelodysplastic syndrome or bone marrow failure: case series with focus on 5-azacytidine and literature review," Eur. $J$. Haematol., vol. 93, no. 3, pp. 247-259, Sep. 2014.

[54] S. Pilorge, L. M. Doleris, F. Dreyfus, and S. Park, "The autoimmune manifestations associated with myelodysplastic syndrome respond to 5-azacytidine: a report on three cases," Br. J. Haematol., vol. 153, no. 5, pp. 664-665, Jun. 2011.

[55] K. Raj, A. Ho, J. D. Creamer, A. W. P. du Vivier, J. R. Salisbury, and G. J. Mufti, "Complete response of deep neutrophilic dermatosis associated with myelodysplastic 
syndrome to 5-azacytidine," Br. J. Dermatol., vol. 156, no. 5, pp. 1039-1041, May 2007.

[56] J.-B. Fraison et al., "Efficacy of Azacitidine in autoimmune and inflammatory disorders associated with myelodysplastic syndromes and chronic myelomonocytic leukemia," Leuk. Res., vol. 43, pp. 13-17, Apr. 2016.

[57] O. C. Goodyear et al., "Azacitidine augments expansion of regulatory T cells after allogeneic stem cell transplantation in patients with acute myeloid leukemia (AML)," Blood, vol. 119, no. 14, pp. 3361-3369, Apr. 2012.

[58] M. K. Poplutz, I. Wessels, L. Rink, and P. Uciechowski, "Regulation of the Interleukin6 gene expression during monocytic differentiation of HL-60 cells by chromatin remodeling and methylation," Immunobiology, vol. 219, no. 8, pp. 619-626, Aug. 2014.

[59] T. Soysal et al., "Bone marrow transplantation for Behçet's disease: a case report and systematic review of the literature," Rheumatology (Oxford), vol. 53, no. 6, pp. 11361141, Jun. 2014.

[60] T. Daikeler et al., "Allogeneic hematopoietic SCT for patients with autoimmune diseases," Bone Marrow Transplant, vol. 44, no. 1, pp. 27-33, Jan. 2009.

[61] R. Billstrom, H. Johansson, B. Johansson, and F. Mitelman, "Immune-mediated complications in patients with myelodysplastic syndromes--clinical and cytogenetic features," European journal of haematology, vol. 55, no. 1, pp. 42-8, Jul. 1995.

[62] F. Bouali et al., "[Immunological abnormalities in myelodysplastic syndromes. Prospective study (series of 40 patients)]," La Revue de medecine interne / fondee ... par la Societe nationale francaise de medecine interne, vol. 26, no. 10, pp. 777-83, Oct. 2005.

\section{Figure and table legends:}

Table 1: Literature studies of ADs associated with MDS. 
$M D S=$ myelodysplastic syndrome, $S L D=$ single lineage dysplasia, $M L D=$ multilineage dysplasia, $E B=$ with excess blasts, $R S=$ with ring sideroblasts, $C M M L=$ chronic myelomonocytic leukemia, $A M L=$ acute myeloid leukemia, $R P=$ relapsing polychondritis, SLE = systemic lupus erythematosus, CTD = connective tissue disease, SS= Sjogren syndrome, $R A=$ rheumatic arthritis, $P R=$ polymyalgia rheumatica, $I H A=$ immune hemolytic anemia, $I T P=$ immune thrombocytopenic purpura, IS = immunosuppressive drugs $(M T X, E d x$, azathioprine), $R / D=$ relapse/ steroids dependence, $N A=$ not available

Table 2: Arguments for MDS-related ADs in patients with extra-hematological features.

Figure 1: Therapeutic strategy for autoimmune disease associated with MDs. ADs = Inflammatory and autoimmune disorders, HMA= Hypomethylating agents 